\title{
A rare case of melanotic hyperpigmentation of the tongue secondary to radiotherapy
}

\author{
Orla A. Houlihan, Guhan Rangaswamy, Orla McArdle \\ St. Luke's Radiation Oncology Network, Dublin, Ireland
}

\begin{abstract}
Melanotic hyperpigmentation of the mucosa secondary to radiotherapy is a rare occurrence. It is a diagnosis of exclusion. Literature review has identified only two case reports published to date. We present a case of a patient treated at our institution. An 18-year-old male patient of Nigerian descent underwent radical radiotherapy ( $36 \mathrm{~Gy}$ in 18 daily fractions) to his right neck for paediatric type follicular lymphoma over a period of four weeks. He developed hyperpigmented tongue lesions during the third week of radiotherapy. There was no associated tongue discomfort, inflammation, infection, or pigmentation change elsewhere in the oral mucosa. Review of medications and past medical history did not demonstrate any potential contributing factors. Full blood count and biochemistry, morning cortisol levels and coagulation screen were all normal apart from mild neutropenia and lymphopenia. His oral cavity received a mean dose of $16.4 \mathrm{~Gy}$, with the right side of his tongue receiving up to $37.5 \mathrm{~Gy}$ as this was within the planning target volume (PTV). He had an excellent response to radiotherapy and remains in remission. The tongue lesions resolved spontaneously 3 months post treatment.
\end{abstract}

Key words: hyperpigmentation; tongue; radiotherapy; lymphoma

Rep Pract Oncol Radiother 2021;26(2):320-323

\section{Introduction}

Hyperpigmented lesions on the tongue are not uncommon and are associated with a wide variety of potential causes. These include physiological [1], medication-induced, such as pegylated interferon, ribavirin [2-4] and chemotherapy [5], endocrine causes, including Addison's disease [6], malignancy such as malignant melanoma [7], and others [8,9]. Radiotherapy is listed as a potential aetiology for this occurrence in only two publications $[10,11]$.

We describe a case report of a patient who developed melanotic hyperpigmentation of the tongue during radiotherapy treatment.

\section{Case report}

An 18-year-old male student of Nigerian descent presented with a lump in his right neck and was subsequently diagnosed with stage II paediatric type follicular lymphoma. He had a diagnosis of epilepsy, although was seizure-free for the previous seven years and cerebral palsy due to pre-term birth. He did not take any regular medications. He was a non-smoker and did not drink alcohol.

At multidisciplinary team discussion, the tumour was felt to be inoperable due to close proximity to neurovascular structures. Chemotherapy was recommended; however, he declined any sys- 


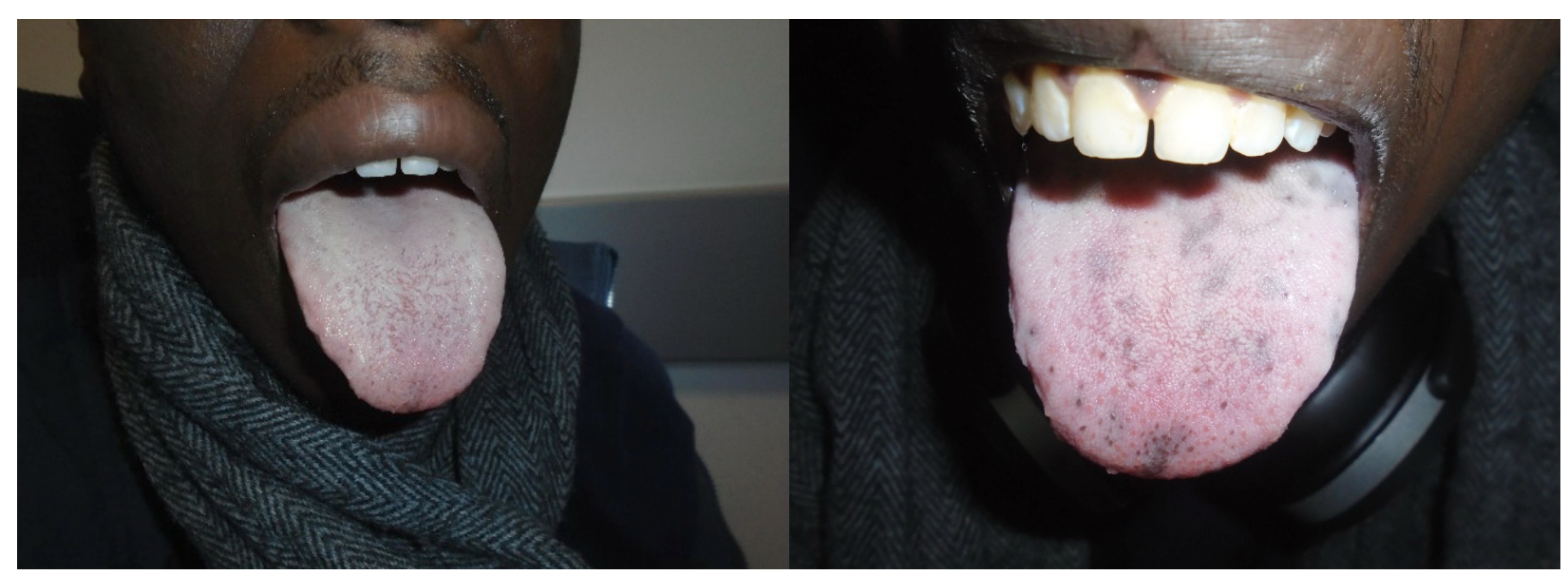

Figure 1. Our patient's tongue during week three of radiotherapy (left) and three months following completion of radiotherapy (right)

temic treatment due to concerns regarding fertility. After much deliberation he agreed to proceed with a course of radical radiotherapy. Physical examination of the neck prior to commencing radiotherapy was significant for a $4.3 \mathrm{~cm} \times 3.4 \mathrm{~cm}$ right neck lump in the submandibular region. Oral cavity examination was unremarkable.

He was treated with fractionated volumetric arc radiotherapy (VMAT), using 6MV photon beams. The gross tumour volume (GTV) was contoured. The clinical target volume (CTV) was delineated according to involved site radiotherapy guidelines with a $15 \mathrm{~mm}$ cranio-caudal margin. A $5 \mathrm{~mm}$ margin was applied to form the planning target volume (PTV), as per our institutional protocol. The prescription dose was 36 Gy in 18 daily fractions, treating five days per week over a period of 4 weeks.

During the third week of radiotherapy he developed hyperpigmented lesions on his oral tongue, as shown in Figure 1. On examination, these dark macules were located on the dorsum and lateral borders of the tongue. The mucosa of the tongue was intact with no signs of infection or inflammation. On palpation, the tongue was non-tender and of normal texture. The rest of the oral cavity was clear, with no signs of mucositis and there were no hyperpigmented lesions elsewhere. The lesions did not cause him any symptoms, specifically, they did not cause him any pain nor did they affect his taste. The radiotherapy plan was reviewed. The dosimetric distribution of radiotherapy delivered to the oral cavity is illustrated in Figure 2. The right posterolateral tongue was included in the PTV, and so the maximum dose received by the tongue was
37.5 Gy. The entire oral cavity received a minimum of $6.8 \mathrm{~Gy}$, while the mean dose delivered was 16.4 Gy. He had no systemic symptoms and otherwise was tolerating radiotherapy very well.

Review of medications and past medical history did not demonstrate any potential aetiology for the hyperpigmented lesions. Full blood count and biochemistry, morning cortisol levels and coagulation screen were all normal apart from mild neutropenia and lymphopenia. A dermatology consult was obtained and we planned to observe the lesions while he remained asymptomatic but to biopsy should he develop any symptoms.

As he was otherwise well we continued his course of radiotherapy as planned with no interruptions. The hyperpigmented lesions began to resolve spontaneously following completion of radiotherapy and had resolved completely by three months (Figure 1). He remains well and cancer-free at 2.5 years post treatment, with no recurrence of the tongue lesions.

\section{Discussion}

This case describes the rare phenomenon of radiotherapy-induced melanotic hyperpigmentation of the tongue. It is a diagnosis of exclusion. Hyperpigmentation of the tongue has been reported in multiple publications, however, the authors could only find two publications which describe this occurrence as a direct result of radiotherapy $[10,11]$.

One paper by Amdur et al. [10] reports on three patients of African American ethnicity undergoing radiotherapy to the brain who developed hyper- 


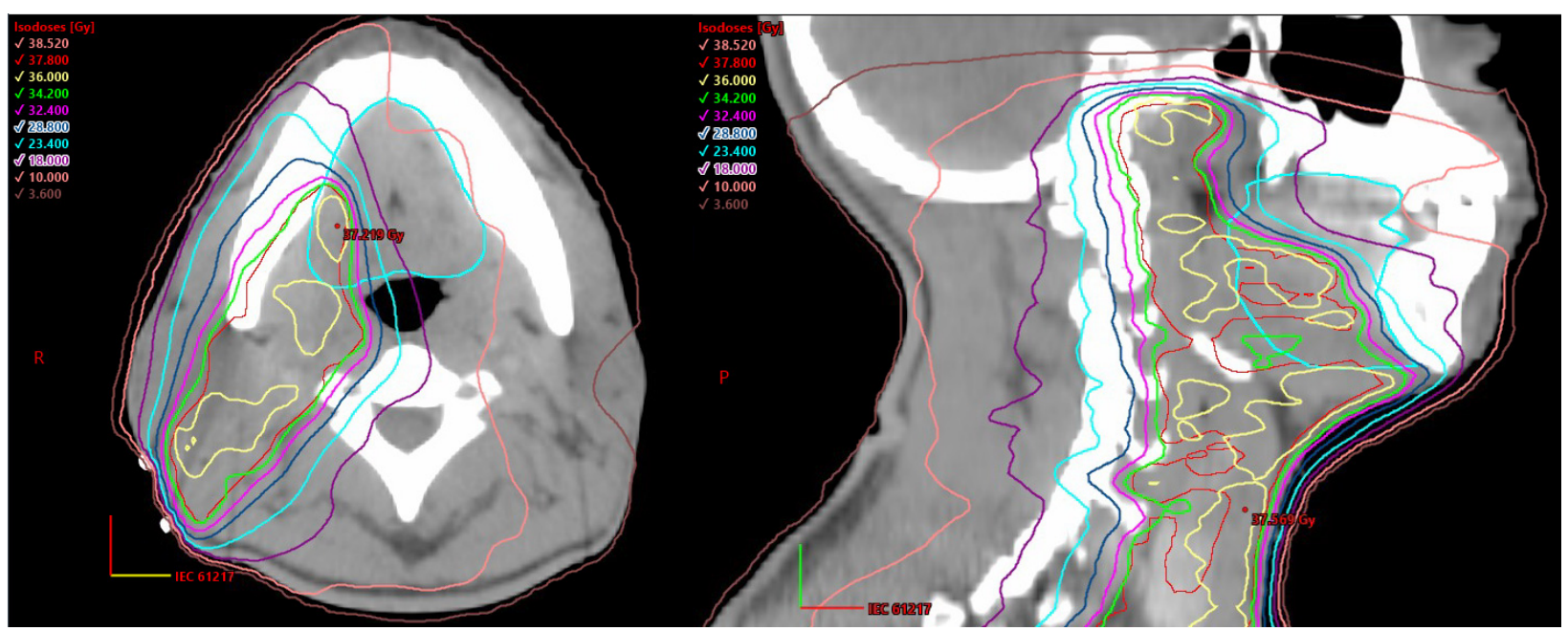

Figure 2. Axial (left) and sagittal (right) dosimetric distribution of radiotherapy delivered to the oral cavity in our patient's radiotherapy plan. Contours: PTV (red), oral cavity (cyan)

pigmentation of the tongue. All patients received low dose radiotherapy to the oral cavity due to exit beam dose. There was no associated mucositis or other oral cavity side effects. The hyperpigmentation was limited to the oral tongue. It developed near the end of the five to six week course, gradually faded after three months and completely resolved by twelve months.

Barrett et al. [11] reported hyperpigmentation of the buccal mucosa and dorsum of the tongue of a Caucasian male. This occurred six months following completion of radical radiotherapy to metastatic carcinoma in the cervical lymph nodes. The oral cavity was included within the target volume for this patient and he experienced side effects of diffuse mucositis, dry mouth and oral discomfort related to the high dose of radiation received. The hyperpigmentation subsequently progressed within a few months to the lateral border of the tongue with hyperkeratosis. Incisional biopsy demonstrated interspersed areas of epithelial atrophy and hyperparakeratosis, candida infection, increased melanin deposition within basal keratinocytes and pigmentary incontinence, in addition to scattered abnormal irradiation fibroblasts but no vascular changes typical of irradiation.

In our patient the dose to the oral cavity and tongue was due to the location of PTV. While the right side of his tongue received a higher radiation dose compared to the left side, the distribution of hyperpigmentation was even throughout the tongue.
The pathophysiology of melanotic hyperpigmentation of the tongue secondary to radiotherapy is unclear; however, it appears to be a temporary phenomenon with spontaneous resolution after a period of months. Melanin is produced in epidermis, hair follicles, cochleae, eyes (choroid, irises, retinal pigment epithelium), heart, lung and brain (leptomeninges, sustantia nigra, locus coerulus) [12]. Its production is not limited to melanocytes and ectopic synthesis in adipose tissue has been reported [13]. Our patient did not have a biopsy, which would have definitively proven the pigmentation was secondary to melanin production. It is unusual that the hyperpigmented macules just appeared in the tongue of our patient, and not other areas where melanocytes are located, including the rest of the oral cavity. This was also the case in the paper by Amdur et al. [10].

Our patient's presentation is similar to that documented by Amdur et al. [10], in terms of ethnicity, timing and anatomical distribution of hyperpigmentation. It is markedly different from that reported by Barrett et al. [11], raising the possibility for differing pathophysiology. Ethnicity may be relevant to our patient's presentation due to the increased activity of melanocytes in African, Asian and Mediterranean populations, which increases the preponderance of physiologic pigmentation in these populations [1, 14]. Barrett et al. [11] suggest that radiation-induced inflammation may cause stimulation of melanocytes, resulting in hyperpigmentation. 


\section{Conclusion}

Melanotic hyperpigmentation of the tongue secondary to radiotherapy is a rare phenomenon; however, it does occur. It is a diagnosis of exclusion and it is essential that affected patients are investigated fully by means of a comprehensive history, clinical examination and laboratory tests, with consideration for biopsy and radiological investigations if clinically appropriate. It is a potential side effect of radiotherapy to the head and neck region, and clinicians should be aware of its existence.

\section{Conflict of interest}

None declared.

\section{Funding}

None declared.

\section{Acknowledgements}

We would like to thank our patient who has consented to the writing of this case report.

\section{Author contributions}

O.A.H., G.R. and O.M. were involved in the care of this patient and wrote the paper. All authors have approved the final article.

\section{References}

1. Chandra S, Keluskar V, Bagewadi A, et al. Extensive physiologic melanin pigmentation on the tongue: An unusual clinical presentation. Contemp Clin Dent. 2010; 1(3): 204-206, doi: 10.4103/0976-237X.73205, indexed in Pubmed: 22114419.

2. Gurguta $C$, Kauer $C$, Bergholz U, et al. Tongue and skin hyperpigmentation during PEG-interferon-alpha/ribavirin therapy in dark-skinned non-Caucasian patients with chronic hepatitis C. Am J Gastroenterol. 2006; 101(1): 197-198, doi: 10.1111/j.1572-0241.2005.00323.x, indexed in Pubmed: 16405555.
3. de Moraes PC, Noce CW, Thomaz LA, et al. Tongue hyperpigmentation resulting from peginterferon alfa and ribavirin combination therapy: a case report. J Am Dent Assoc. 2009; 140(11): 1377-1379, doi: 10.14219/jada. archive.2009.0073, indexed in Pubmed: 19884395.

4. Willems M, Munte K, Vrolijk JM, et al. Hyperpigmentation during interferon-alpha therapy for chronic hepatitis C virus infection. Br J Dermatol. 2003; 149(2): 390-394, doi: 10.1046/j.1365-2133.2003.05422.x, indexed in Pubmed: 12932249.

5. Stringer LL, Zitella L. Hyperpigmentation of the tongue. J Adv Pract Oncol. 2014; 5(1): 71-72, indexed in Pubmed: 25032039.

6. Sarkar SB, Sarkar S, Ghosh S, et al. Addison's disease. Contemp Clin Dent. 2012; 3(4): 484-486, doi: 10.4103/0976237X.107450, indexed in Pubmed: 23633816.

7. Rowland HN, Schnetler JF. Primary malignant melanoma arising in the dorsum of the tongue. Br J Oral Maxillofac Surg. 2003; 41(3): 197-198, doi: 10.1016/s02664356(03)00025-1, indexed in Pubmed: 12804549.

8. Kauzman A, Pavone M, Blanas N, et al. Pigmented lesions of the oral cavity: review, differential diagnosis, and case presentations. J Can Dent Assoc. 2004; 70(10): 682-683, indexed in Pubmed: 15530266.

9. Meleti M, Vescovi P, Mooi WJ, et al. Pigmented lesions of the oral mucosa and perioral tissues: a flow-chart for the diagnosis and some recommendations for the management. Oral Surg Oral Med Oral Pathol Oral Radiol Endod. 2008; 105(5):606-616, doi: 10.1016/j.tripleo.2007.07.047, indexed in Pubmed: 18206403.

10. Amdur RJ, Sandow PR, Yeung A, et al. Melanocytic hyperpigmentation of the tongue from low-dose radiotherapy. J Hong Kong Coll Radiol. 2010; 13: 32-5.

11. Barrett AW, Porter SR, Scully C, et al. Oral melanotic macules that develop after radiation therapy. Oral Surg Oral Med Oral Pathol. 1994; 77(4): 431-434, doi: 10.1016/00304220(94)90209-7, indexed in Pubmed: 8015811.

12. Brenner M, Hearing VJ. What are melanocytes really doing all day long...? : from the viewpoint of a keratinocyte: melanocytes - cells with a secret identity and incomparable abilities. Exp Dermatol. 2009; 18(9): 800-802.

13. Randhawa M, Huff T, Valencia JC, et al. Evidence for the ectopic synthesis of melanin in human adipose tissue. FASEB J. 2009; 23(3): 835-843, doi: 10.1096/fj.08-116327, indexed in Pubmed: 18971261.

14. Gaeta GM, Satriano RA, Baroni A. Oral pigmented lesions. Clin Dermatol. 2002; 20(3): 286-288, doi: 10.1016/s0738081x(02)00225-0, indexed in Pubmed: 12074869. 